\title{
No presentism in quantum gravity
}

\author{
Christian Wüthrich \\ To be published in Vesselin Petkov (ed.), Space, Time, and Spacetime: Physical and \\ Philosophical Implications of Minkowski's Unification of Space and Time, Springer, 2010.
}

\begin{abstract}
This essay offers a reaction to the recent resurgence of presentism in the philosophy of time. What is of particular interest in this renaissance is that a number of recent arguments supporting presentism are crafted in an untypically naturalistic vein, breathing new life into a metaphysics of time with a bad track record of co-habitation with modern physics. Against this trend, the present essay argues that the pressure on presentism exerted by special relativity and its core lesson of Lorentz symmetry cannot easily be shirked. A categorization of presentist responses to this pressure is offered. As a case in point, I analyze a recent argument by Monton (2006) presenting a case for the compatibility of presentism with quantum gravity. Monton claims that this compatibility arises because there are quantum theories of gravity that use fixed foliations of spacetime and that such fixed foliations provide a natural home for a metaphysically robust notion of the present. A careful analysis leaves Monton's argument wanting. In sum, the prospects of presentism to be alleviated from the stress applied by fundamental physics are faint.
\end{abstract}

\section{Introduction}

Presentism is the position in the philosophy of time that maintains that nothing exists that is not present. In other words, only present events and objects exist, but no past or future events or objects do. Furthermore, it usually assumes that there is a succession of presents, i.e. a moving Now. Although logically independent from the thesis that defines the position, most presentists thus take change, or becoming, to be a fundamental aspect of reality. Bradley Monton (2006, 264) has appropriately dubbed the package of presentism-cum-becoming "Heraclitean presentism". In logical space, as he rightly notes, there could also be a presentist metaphysics which holds that the spatially extended sum total of existence is completely static in that fundamentally, it does not involve change at all. Such a "Parmenidean" version of presentism, however, has rarely, if ever, been entertained 1 What is of relevance to my present purposes is simply the core thesis of presentism according to which only present events and objects exist, and not whether this core is adorned with Heraclitean or Parmenidean plumes.2

\footnotetext{
${ }^{1}$ Barbour (1999) can be read as offering a Parmenidean presentist view. Of course, there is lots more logical space available, e.g. containing a presentist position which subscribes to a moving Now without there being any change whatever. Furthermore, the basic presentist claim can be read as obtaining by necessity or merely contingently, which opens logical space for necessitarian and Humean brands of presentism. All these further varieties and distinctions, however, do not affect the present argument. I shall thus ignore them here.

${ }^{2}$ I understand that there is real worry about whether the debate between presentism and eternalism is well-formed and metaphysically substantive, cf. Callender (2000), Dorato (2006), and Savitt (2006a). As I argue in an unpublished essay, however, I believe that these worries can ultimately be dispelled. I wish to thank Steve Savitt for taking me to task on this issue.
} 
There are a number of metaphysical objections against presentism in the literature, and they shall not be surveyed here. Moreover, some authors have denied that it presents the only, or even best, way to account for our intuitions about the phenomenology of temporality-traditionally considered the strong suit of presentism. But a much more powerful, and potentially devastating, challenge arises from modern physics: Einstein's special relativity (SR) provides strong, and perhaps conclusive, reason to view space and time not as two separable and quite distinct animals, but much rather as entangled aspects of the same underlying four-dimensional manifold that fuses the two into a "spacetime". It was Hermann Minkowski's great achievement to recognize the inseparability of space and time resulting from Einstein's theory when he solemnly declared at the Assembly of German Natural Scientists and Physicians in Cologne in September 1908: "The views of space and time which I wish to lay before you have sprung from the soil of experimental physics, and therein lies their strength. They are radical. Henceforth space by itself, and time by itself, are doomed to fade away into mere shadows, and only a kind of union of the two will preserve an independent reality." Minkowski was also the first to correctly describe the geometrical properties of this fused "space-time" structure that today we call Minkowski spacetime. Section2 2 explicates how SR and its attendant Minkowski spacetime exert significant pressure on presentist positions and thus revisits the issue of compatibility of SR and presentism.

Although SR does not apodictically rule out presentism, it constrains it in a way that renders whatever presentism survives the relativistic revolution a metaphysically rather unattractive cripple. One might have expected that this would do it. But presentism dies hard, very hard. In fact, after a period of relative tranquility, it enjoys something of a renaissance in the philosophy of time. What is striking about this renaissance is that many of the hold-out (or born-again) presentists attempt to support their position by arguments of the kind that have traditionally been the weapon of choice for many of their opponents: arguments drawing on results from the physical sciences. Section 3 analyzes in some detail a particularly interesting case recently offered by Monton (op. cit.). His proposal is important in that it promises to breathe new, scientifically sophisticated life into the otherwise moribund idea of presentism. Section 4 then investigates the prospects of presentism in the so-called constant-mean-curvature $(C M C)$ foliation approach to quantizing gravity, which Monton finds particularly amenable to his presentist inclinations. It will illustrate the many ways in which the CMC approach fails to vindicate presentism, despite its initial allure to the presentist. SR, while strictly speaking false of the actual world, at least in an unqualified sense, imposes an important constraint on feasible physical theories, or at least on all physically acceptable interactions. In this sense, it can also be considered a "second-order theory". This section, it should be warned, will be somewhat technical due to the nature of the material covered in it. Finally, Section 5 offers some conclusions.

\section{Minkowski spacetime and the pressure from special relativity}

The eternalist considers the four-dimensional "block universe" with all of spacetime and everything it contains to make up the sum total of existence. By contrast, the presentist maintains that the sum total of existence can be understood as consisting of a three-dimensional manifold of spatially distinct but temporally equally present, and thus simultaneous, events or objects. Presentism thus seems to require an objective "foliation" of Minkowski's spacetime into hyperspaces of threedimensional "space" ordered by a one-dimensional "time" parameter ${ }^{3}$ In that it claims a different

\footnotetext{
${ }^{3}$ A foliation slices up the four-dimensional spacetime into space and time via an equivalence relation interpreted as "simultaneity". A binary relation $R x y$ is an equivalence relation on a set $S$ iff it is reflexive (for all $x \in S, R x x$ ), symmetrical (for all $x, y \in S$, if $R x y$, then $R y x$ ), and transitive (for all $x, y, z \in S$, if $R x y$ and $R y z$, then $R x z$ ). Space
} 
ontological status for those things present from those non-present, it (usually) presupposes that the distinction between the present and the non-present can be drawn in a principled, objective way. In other words, it requires a metaphysically robust, objectively valid concept of a spatially extended present. ${ }^{4}$ Alas, SR provides a strong reason to believe that that can't be had $!^{5}$

In pre-relativistic physics, the notion of simultaneity of spatially distant events was unproblematic. In SR, however, it turned out that the requisite four-dimensional spacetime had a radically different structure: whether or not two spatially distant events are simultaneous was no longer an objectively and universally determinable fact of the matter. Two inertial observers at some relative velocity with respect to one another do not agree whether two events are simultaneous or not. The relation of simultaneity is thus relativized to reference frames. In a technical language, this means that there is no preferred foliation of spacetime into slices of three-dimensional spaces representing classes of simultaneous events. If we define "the present" as consisting of all those events which occur simultaneous with the point in spacetime representing the here and now, then the relativity of simultaneity seems to imply that the presentist is committed to relativize existence analogously: if we are two inertial observers moving at some relative speed, we take different distant events to be real!

Let's back up a little and have a closer look at how (classical and relativistic) physics conceives of time. Classical Newtonian mechanics does not postulate a Now, but is blatantly compatible with a metaphysically robust and objectively valid concept of a spatially extended present. In fact, a (non-relativistic) time-reparametrization-invariant theory, i.e. a theory in which the action remains invariant under redefinitions of time $t^{\prime}=f(t)$, generally allows for the possibility of an objective spatially extended present, and even for temporal flux or becoming. In such a theory, two situations differing only in their parametrizations of time are really descriptions of one and the same physical situation. Consequently, time does not exist as an objectively measurable independent degree of freedom; more precisely, time is not a magnitude with an objectively privileged metric. In a theory like this, however, there exists an objective total ordering of events in time 6

Special-relativistic theories admit only a partial temporal ordering of events. The loss of absolute simultaneity leads to a loss of comparability: with an interpretation of the binary ordering relation as "being earlier than or simultaneous to" - it is a temporal ordering that we are seeking after all-, pairs of spacelike related events do not stand in this relation. There is simply no frame-independent fact of the matter as to whether event $a$ is earlier than event $b$ or the other way around for two spacelike related events $a$ and $b$. In general-relativistic theories, where the topology of a spacetime may fail to even permit a non-unique foliation of spacetime into space and time, the possibility of

at a time is then given by the corresponding three-dimensional "folium" and time is the one-dimensional linearly ordered quotient set induced by the equivalence relation, "lining up" the moments of simultaneity.

${ }^{4}$ At least standardly; Harrington (2008) has defended a "point present", a radically solipsistic version of presentism according to which not only only temporally present events exist, but also only spatially present ones. For the point presentist, not even all of my present brain exists. Harrington's position evades the objection raised in this sectionbut at what price!

${ }^{5}$ While this paper focuses on presentism, a possibilist metaphysics defending a growing block or branching tree structure faces analogous challenges from SR. For instance, McCall (2000) maintains the reality of the past and the present, with the future as a branching set of four-dimensional alternatives. The "present" is the first branch surface, which is defined as a maximal set of pairwise spatially separated events. In order to uphold Lorentz invariance, the branch attrition along these surfaces is relativized to inertial frames. In this sense, McCall's view is the possibilist analogue of Fine's presentism, presented below.

${ }^{6}$ A total order on a set $S$ is given by a binary relation $R$ that is reflexive ( $R a a$ for all $a$ in $S$ ), weakly antisymmetrical (for all $a, b \in S, R a b$ and Rba entails $a=b$ ), transitive (for all $a, b, c \in S, R a b$ and $R b c$ entails Rac), and comparable (for any $a, b \in S$, either $R a b$ or $R b a$ ). A partial order on a set is a binary relation with the first three properties, but not the last one. Thus, in a partially ordered set, there exist pairs of elements in the set which do not exemplify the relation. 
causal loops entails that the temporal ordering is in general not even weakly asymmetric, i.e. there no longer is a partial temporal order of events. In fact, there is no global time deserving this title in general relativity (GR), a fact that finds a particularly vivid expression in the so-called "problem of time" arising in the Hamiltonian formulation of GR. Sic transit gloria temporis.

Let's see what all of this implies for the prospects of presentism. Suppose one upholds the following basic commitments:

Naturalism: Our metaphysical positions must be compatible with physics, at least to the extent to which the latter is taken to be true of the world.

SR-Realism: Special relativity (SR) is taken to be (approximately) true of the world.

Presentism: There exists an objective spatially extended present and only events or objects in this present exist.

Of course, Presentism implicitly asserts that it is a coherent, non-trivial, substantive metaphysical position. Naturalism and SR-Realism jointly imply

Compatibilism: Whatever metaphysical view of the world we advance must be compatible with the fact that SR is (approximately) true.

My purpose here is not to defend any of these theses but only to ask whether a commitment to Compatibilism is consistent with maintaining Presentism. It is, as we shall see. The question, however, is whether Compatibilism leaves the presentist with an interesting position at all. The lesson gleaned from an argument independently advanced by Wim Rietdijk (1966) and Hilary Putnam (1967) suggests that it does not. Since their argument is well known, let me only briefly remind the reader how it essentially goes $]^{7}$

The Rietdijk-Putnam argument assumes that the task is to figure out which of the spatially distant events in the four-dimensional spacetime are co-present with the here-now. To identify the objective, spatially extended present strikes me as an unavoidable task if presentism is characterized as I did above. Next, introduce an equivalence relation $R$ interpreted as "being simultaneous with". Then, use $R$ to construct the spatially extended present, starting out from the here-now. The problem essentially is, as mentioned above, that in SR simultaneity relations become framerelative. This was the content of the relativity of simultaneity. If in Figure 1, $e$ designates the here-now, then the event denoted by $a$ is simultaneous to $e$ as far as the primed frame is concerned, but in the future of $e$ according to the unprimed frame. In other words, in the primed frame, Rae, but in the unprimed frame, $\neg$ Rae. Thus, there is no objective fact of the matter which spatially distant events are co-present with the here-now. It gets worse. Since simultaneity is a transitive relation, one would expect that what is co-present with a spatially distant event co-present with the here-now is also co-present with the here-now. Consider the situation as shown in Figure 2. In the unprimed frame, $e^{\prime}$ is certainly simultaneous with $e$ and because $e$ represents the here-now, $e^{\prime}$ is also present (and thus exists). However, in the primed frame $b$ is certainly simultaneous with $e^{\prime}$ and because $e^{\prime}$ is present, $b$ is also present (and thus exists). Moving from one frame of reference to another in the course of the argument ought to be acceptable if simultaneity were objective, i.e. frame-independent. Of course in SR, it isn't. But that's the point. Consequently, a presentist is committed to the existence of event $b$ which is in the future of $e$ with respect to all frames of reference. But this is surely a reductio of the position.

Presentists have responded in a variety of ways to the pressure exerted by the Rietdijk-Putnam argument and I shall not list them in any detail, but just highlight the basic strategic options. Here

\footnotetext{
${ }^{7}$ For a more basic and detailed rendering, see Savitt (2006b).
} 


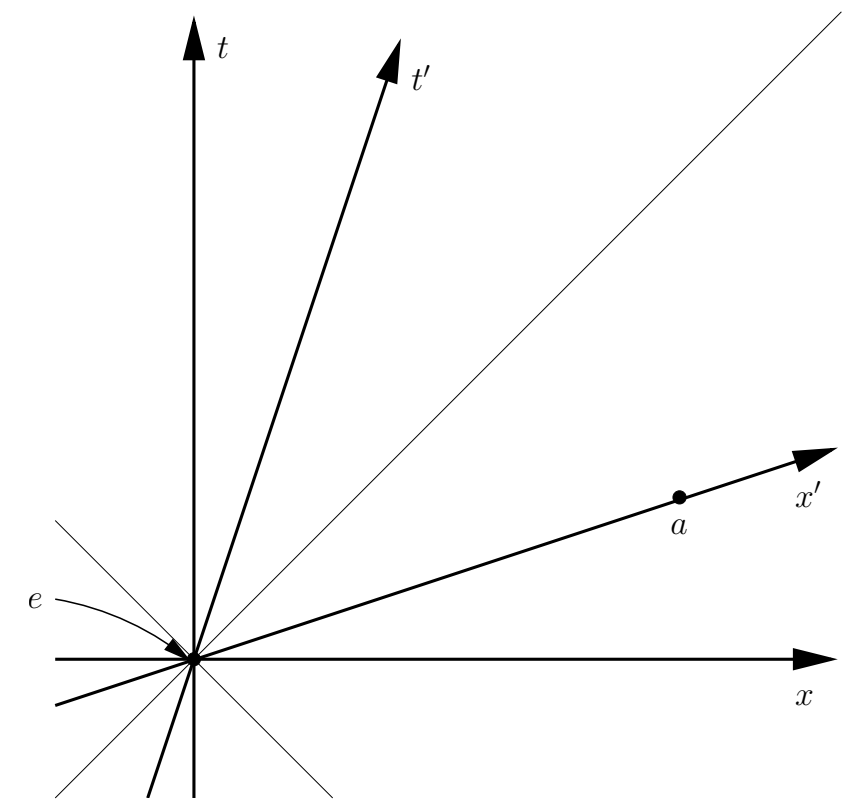

Figure 1: The Rietdijk-Putnam argument illustrated.

are some incompatibilist strategies, i.e. responses rejecting Compatibilism in one form or other. First, a presentist could deny Naturalism. Such denial could take different forms. One could, as does Jonathan Lowe ${ }^{8}$ claim that SR is not a theory about time but about something else instead. Alternatively, one could retort by accepting that SR speaks to the geometry of spacetime but reject that this has any ontological import, as does Dean Zimmerman (2008) 9 $^{9}$ Second, a presentist might reject SR-Realism, simply asserting that SR is not approximately true of the world. This could occur simply on a priori grounds, an option I will not comment on. In fact, the remainder of this essay after this section will be dedicated to explore a posteriori exit strategies denying SR-Realism. Relevantly, Monton (op. cit.) can be read as a representative of this strategy, as will become clear below. Also, considerations from quantum mechanics can be invoked in an attempt to establish that SR is false or incomplete insofar as it lacks an absolute, privileged frame of reference. This response comes in different flavours: (a) (non-relativistic) collapse dynamics require a preferred frame in which the collapse occurs; (b) Bohmian interpretations are incompatible with SR; and (c) invoke Bell's theorem to argue that some tenets of SR must be given up. I concur with Craig Callender (2008) that these strategies don't succeed, but will not elaborate here.

What are the basic compatibilist responses at the presentist's disposal? First, the set-up of the Rietdijk-Putnam argument could be rejected as doing violence to a genuinely presentist metaphysics. What is more or less tacitly presupposed in the argument, it could be insisted, viz. that there is a four-dimensional manifold of spacetime events such as Minkowski spacetime of which it

\footnotetext{
${ }^{8}$ In a paper entitled "Experience of change and change of experience", delivered at the University of Geneva on 19 December 2008.

${ }^{9}$ Zimmerman, together with a number of present-day presentists, is hard to classify as either compatibilist or incompatibilist as he accepts SR, but not in the role a naturalist usually would. He thinks that SR leaves room for an additional relation of simultaneity not to be found in physics. This relation would only clash with physics if the latter were committed to a principle prohibiting extra relations of this sort, but such a principle, he thinks, would not be warranted. Of course, this relation would still effectively foliate spacetime. Such a foliation could either be observed, or it couldn't. If the former, Compatibilism would be denied; if the latter, we run into similar problems as the defense championed by Tooley and Craig, which is essentially of that type and shall be discussed below. I thank Jonathan Cohen for having reminded me of this connection.
} 


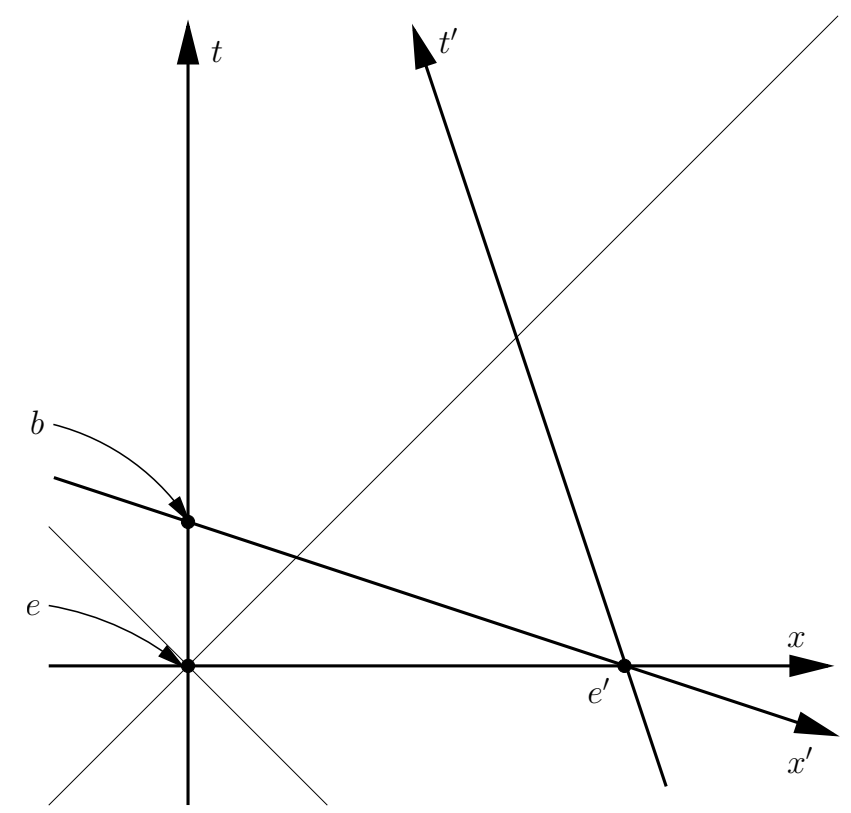

Figure 2: An event to the future of the here-now (in any frame) is co-present with it.

is then our task to determine which of these events are "determinate" as of the here-now or are objectively present, ought to be discarded by the presentist. While I think that it is still a perfectly justifiable task to ask of the presentist to describe the sum total of existence, to somehow tell a story as to how her position can be reconciled with SR, the Rietdijk-Putnam argument certainly still has force against an ersatzist version of presentism, which, as I have argued elsewhere (unpublished), we are forced into in order to save presentism from the threat of trivialization. On the other hand, a presentist might simply bite the bullet and consequently relativize existence, an option chosen by Kit Fine (2005; particularly $\S 10$, pp. 298-307): since what is present is relative to an inertial frame, what exists becomes fragmented in that it depends on the choice of frame. There is an intermediate strategy, somewhere between accepting the full consequences of the argument and rejecting the way it sets up the presentist commitments: define the objectively existing present purely in terms of the Lorentz-invariant structure available in Minkowski spacetime. The solipsistic version mentioned earlier (in footnote 4) and defended by James Harrington (2008) trivially makes only use of the Lorentz-invariant structure, viz. a single spacetime point as representing the spatiotemporal location of the sum total of existence. But does this capture the true spirit of presentism? It can be doubted, as neither existence nor "becoming" remain universal on this proposal. Fine (2005, 304) puts it succinctly: presentists tend to be impressed by the distinction between space and time which they take to be metaphysically deep in that they think that there exists an objective "now", although there does not exist an equally objective "here". Of course, this intuition is lost in solipsistic presentism. Accordingly, it violates Presentism as I defined it above.

An alternative way of make exclusive use of the Lorentz-invariant structure has been proposed by Howard Stein (1991) and could be termed past-light cone presentism. The main idea is to identify the spatially extended present as the set of events on the past light cone of the here-now. Yes, you haven't misread: the idea is to define the present as the set of events on the past light cone. This proposal is Lorentz-invariant and can be motivated by an appreciation of epistemic accessibility, as causal signals reaching us now emanate from the events on the past light cone and thus appear to us as being co-present. While on the solipsistic version, the simultaneity relation remains, trivially, 
an equivalence relation, it is no longer symmetrical and transitive in past-light cone presentism. Symmetry, but not transitivity, can be restored by extending existence to events on the future light cone. But in what sense would this still be the present? Points on Andromeda some four million years apart in time, but at no distance in space according to some joint frame of reference for a generic observer on earth and one on Andromeda, would both be co-present with the here-now.

A final compatibilist strategy that ought to be mentioned is to accept that SR offers a perfectly empirically adequate theory, but to insist that absolute simultaneity still exists. It is just that we cannot possibly detect the privileged frame of reference which determines the present. In other words, absolute simultaneity is not empirically accessible. This strategy is, arguably, compatibilist only in letter, but not in spirit. Its motivations may be metaphysical or physical. A variant of the former is found in Michael Tooley (1997), one of the latter in neo-Lorentzian interpretations of SR, such as the one attempted by William Craig (2001) 10 In both cases, the metaphysics fully relies on postulated extra-structure that can't even in principle be observed. The extra-structure needed is not motivated by more than specific metaphysical agendas or a refusnik attitude toward SR. It violates Ockham's razor so crassly that the move cannot be justified by putting some postverificationist philosophy of science on one's flag. An argument to the effect that since it is only because of some ill-advised verificationist commitment that SR prohibits a privileged frame, and since we know that verificationism is false, we can infer that there is absolute simultaneity, does obviously not succeed. But note that even if we permitted the stipulation of this unobservable extra-structure, such as a simultaneity relation, it appears that it cannot do the work asked of it. If one's goal is to produce a metaphysics that vindicates our pre-theoretical (non-)ascriptions of simultaneity, then a postulated simultaneity relation will not help such vindication so long as it is epistemically inaccessible. And if it is epistemically accessible, Compatibilism is violated even in letter.

In sum, the prospects of compatibilist strategies appear bleak. Those of incompatibilist responses hardly seem brighter, at least not for those of us who accept Naturalism - except if we came to offer a strong a posteriori argument as to why SR does not approximately hold of the actual world. There is plenty of physics that such an argument could turn on. It could be that Lorentz symmetry only holds approximately and at large scales, e.g. if the underlying spacetime structure is discrete. Depending on how approximately it would hold, this may still lead to a compatibilist strategy. It could be that if gravity is turned on, or if we take quantum effects into considerations, or both, it will be seen that SR is invalid. To discuss, or even list, all the physics that such an argument could make use of is the task for another day. It is an interesting task that will lead the investigator into a thick, and almost impenetrable, forest of foundational issues in fundamental physics. Today, I will confine myself to an analysis of the suggestion in this vein recently made by Monton (op. cit.).

\section{Monton's incompatibilist defence of presentism}

Monton sums up the Rietdijk-Putnam argument as follows (op. cit., 264):

(1) "Presentism is incompatible with [special] relativity [...]"

(2) SR "is our most fundamental theory of physics."

(3) "Presentism is incompatible with our most fundamental theory of physics (from (1) and (2))."

\footnotetext{
${ }^{10}$ Craig also seems to think that SR is a kinematic theory that only underwrites electrodynamics, and not all or even most of physics. This is simply false. Physicists are working hard to make sure that all theories are Lorentz-invariant. If they fail in doing so, it is generally accepted that their theory faces a major problem.
} 
While Monton recognizes that the step from (3) to (4) is non-trivial, he finds it preferable if the presentist wouldn't have to rely on blocking that step. In other words, at least for the sake of the present argument, he accepts Naturalism. Consequently, rejecting the argument will require denying either one of the first two premises or the inference from them to (3). But this inference is obviously valid. Offering an incompatibilist stance, Monton accepts premise (1). Remains premise (2): Monton finds it "relatively uncontroversial" that thesis (2) is false, i.e. that SR is not our most fundamental theory of physics. There is certainly a sense in which he is right: once gravity is taken into account, SR must be replaced by GR which is arguably more fundamental; GR is incompatible with quantum physics and both must be superceded by a quantum theory of gravity, which in turn may ultimately be supplanted by a "theory of everything". Thus, (2) is false. Of course, (3) could still be true, viz. exactly in those cases where it turns out that the final fundamental theory of physics is still incompatible with presentism, perhaps for reasons unrelated to the relativity of simultaneity. But, injects Monton, there are quantum theories of gravity which are compatible with presentism. What he has in mind here are approaches in so-called fixed-foliation quantum gravity (QG), such as QG relying on foliations of spacetime into hypersurfaces of constant mean (extrinsic) curvature or "CMC" for short. From the existence of such theories in QG, he infers that "(3) is false, and presentism is unrefuted" (ibid., 265). This inference is of course only valid if it is the case that one of those quantum theories of gravity compatible with presentism is in fact the most fundamental theory of physics. I will overlook, at least for now, this overly excited inferential step, but we will have to revisit it.

Monton's argument can be thought of as consisting of two steps: first, SR is marginalized as an irrelevant, and false, theory; second, the CMC approach to QG is then presented to add credence to the claim that fundamental physics is hospitable to presentism. The remainder of this section discusses the first part of the argument, the next section analyzes the second part.

Let me give three preliminary comments. First, I find it rather curious that Monton formulates the argument in terms of which theories are fundamental. Whether or not a theory - any theorywith which presentism's compatibility is tested is fundamental or not seems entirely beside the point. What matters is truth. Incompatibility with a theory which is true of the actual world seems a sufficient condition to rule out a metaphysical proposal. Presumably, fundamentality entails truth; no theory could reasonably be considered fundamental if it were not true. But of course fundamentality is not necessary for truth. There are many theories about higher-level phenomena, and presumably some of them are true without being fundamental. But that's the crux: by denying that SR is fundamental, Monton means to imply that it is false. Since incompatibility with a false theory is not problematic, presentism would be saved. In general, however, non-fundamentality does not entail falsehood. The situation at stake is more subtle, as it turns out. Strictly speaking, and if no qualifications about its domain of applicability are added, SR is a false theory: it is not in toto true of the actual world. However, it is still believed to impose a very rigid constraint on any candidate fundamental theory. Just exactly what this constraint is will ultimately be decisive in adjudicating whether presentism is compatible with the best physical theories true of our actual world. I will return to this below.

In a sense, it's even worse than this. Arguably, fundamentality imposes a partial ordering on theories. But this means that there may fail to be a fact of the matter as to which one of two particular theories is more fundamental. Furthermore, fundamentality may not be well-defined or philosophically justifiable as an important, or relevant, criterion. Thus, fundamentality appears to be a requirement which may be inapplicable in, as well as irrelevant to, the case at hand. 
Second, let me illustrate the dialectical landscape as I see it. We have seen above that Monton's argument can only offer respite for presentism if it cannot only claim that SR is not a fundamental theory, but if it can also be made credible that presentism has good chances of being compatible with our most fundamental theory of physics, and that (3) is thus false. But in order to establish that, Monton must navigate between the Scylla of triviality and the Charybdis of falsehood. On the one hand, his argument could be interpreted as primarily expressing general scepticism about the current state of physics. Since we don't have the physics in the ideal limit of scientific enquiry, would be the thought, a presentist can maintain hope that she will ultimately be vindicated. But such a hope would be pious indeed. Thus, if the intended conclusion is simply that in principle it could be that presentism will eventually be compatible with fundamental physics, then it is disappointingly trivial.

On the other hand, Monton's argument may be read as offering a crystal ball from which the future of QG can be gleaned. Here, the idea would be to reach a prediction that, at least with reasonable probability, the final theory will be hospitable to presentism. But such a prediction would be audacious indeed. In fact, if the claim is that it is reasonably likely that presentism will eventually be compatible with fundamental physics, then the argument is unacceptably false.

It might be protested that I am striking Monton with an unfair dilemma. I am not: I don't claim that his conlusions are either trivial or false. What I am saying, however, is that he must strike a fine balance in order to end up with a substantive and true conclusion. What I will attempt to show in much of the remainder of this essay is that the room to manoeuvre between said Scylla and Charybdis is uncomfortably tight.

Third, Monton treats the classical and the corresponding quantum version of a theory curiously disanalogous. Such a disparity may sometimes be justified, but arguably not here. Let me explain. Monton seems to think that choosing a particular (CMC) foliation is inadmissible at the classical level, but entirely unproblematic once we go to the quantum theory. He asserts that presentism is incompatible with SR and GR because Minkowski and general-relativistic "spacetimes do not have a foliation into spacelike hypersurfaces as part of their structure." (ibid., 267) Such foliation, he admits, can sometimes be picked out, but "the foliation is not part of the spacetime structure as given, and thus imposing such a foliation amounts to changing the theory." (ibid., 268) It is somewhat mysterious why he has such qualms about changing the theory, particularly since at the end of his essay, he has no hesitation to proclaim that a committed presentist ought to demand that since string theory and loop quantum gravity do not account for presentist intuitions, they ought to be modified accordingly. Furthermore, as will become clear in Section 4 , almost all of the work on the CMC approach has been done at the classical, not at the quantum, level. For canonical approaches, the classical and the quantum levels are not interpretationally independent: canonical quantization necessitates an interpretation of the classical theory to be quantized which will then be carried over into the corresponding quantum theory. Thus, for the fixed-foliation approach to QG that Monton advocates, a CMC interpretation of the classical theory is presupposed and the disparity assumed by Monton seems ill-justified.

Leaving the preliminaries, Monton starts out stating this argumentative goal:

[Because special and general relativity are not our most fundamental theories of physics], the compatibility of presentism with special and general relativity is prima facie irrelevant to the issue of presentism ${ }^{5}$. I will argue that this prima facie appearance is in fact correct. (ibid., 267)

Footnote 5 takes no prisoners: against Mark Hinchliff who asserted that SR is "one of our bestconfirmed scientific theories of the nature of time" (1996, 131), Monton declares that 
[t]his claim is false: the special theory is a decisively refuted theory of the nature of time. Special relativity is incompatible with such phenomena as the gravitational redshift and gravitational lensing, phenomena that provide evidence for general relativity. (ibid.)

As Monton acknowledges, scientists do not reject all old ideas in a scientific revolution. Thus, one might require that the incompatibility of presentism with SR be carried over to any legitimate candidate fundamental theory. However, he quickly dismisses this answer on the basis that since there are many potentially viable approaches to QG, some of which frustrate the demanded incompatibility, there are no compelling grounds on which a presentist must concede an eventual incompatibility.

Because of the lack of data to back up the claim that a good theory is incompatible with presentism,

Monton concludes that

all the literature on the issue of whether presentism is compatible with [...] relativity is [...] irrelevant to the issue of whether presentism is true. (ibid., 269)

While it is certainly true that there is no empirical data directly suggesting an incompatibility with presentism, this conclusion can't be had that easily. The Principle of Relativity, i.e. the demand that the physics is the same in all inertial frames, is encoded in a theory as the Lorentz covariance of its dynamical equations, which means that there can't be any dynamical phenomena that would allow us to pick a privileged frame and thus an absolute simultaneity. In SR, this dynamical symmetry is carried over into the spacetime structure, leading to the geometry of Minkowski spacetime, which of course is invariant under Lorentz transformations. In GR, the Principle of Equivalence ascertains that at each point of spacetime, the spacetime structure exhibits the same symmetry. Quantum field theory (QFT) assumes the Minkowski spacetime as Lorentz-invariant background structure, and QFT on curved spacetime makes the same symmetry assumption for each point of the (curved) spacetime background. In fact, most physicists would agree that dynamical equations ought to be Lorentz-covariant and that the background spacetime at least of semi-classical theories must have the relevant symmetry at least in some local sense.

The fixed-foliation quantum theories of gravity to be discussed in the next section violate the Principle of Relativity in that they require a preferred frame of reference ${ }^{11}$ There is to date, of course, no empirical indication whatsoever that such a preferred frame of reference exists. In fact, Lorentz (or, more precisely, Poincaré) symmetry is fantastically well confirmed ${ }^{12}$ Thus, to require that Lorentz symmetry be valid is well justified. Now, this in itself does not entail an incompatibility of presentism with empirical data. As we have seen in Section 2 , there are perfectly Lorentz-invariant ways of formulating a presentist position, although there is considerable doubt whether they succeed in fully capturing the spirit of presentism. Be this as it may, the fact that Lorentz symmetry is so well confirmed puts serious pressure on any approach that requires a preferred reference frame 13

\footnotetext{
${ }^{11}$ Monton agrees: "the proponent of fixed foliation quantum gravity will agree that there is a preferred frame of reference, and can admit that [...] the theory makes sense only in one reference frame." (ibid., 271)

${ }^{12}$ For an authoritative review of experimental tests of Lorentz symmetry, cf. Will (2005a, b); for a recent review on phenomenological indications that Lorentz symmetry may be broken at the Planck scale, cf. Amelino-Camelia (2008).

${ }^{13}$ Monton addresses remarks by Gordon Belot and John Earman (2001) that could be framed as an objection to his view. They argue that fixed-foliation approaches to QG have few adherents because "[t]o forsake the conventional reading of general covariance as ruling out the existence of preferred co-ordinate systems is to abandon one of the
} 
Let me frame this in more general terms. SR can be thought of as a "first-order theory", i.e. a theory which makes claims about the world and as such can be true or false of the actual world. As it completely ignores gravity, a strong case can be made that it is, in fact, false. However, it might also be regarded as a "second-order theory", i.e. a theory that places certain constraints on other theories. More specifically, it requires that all possible physical interactions be governed by Lorentz-covariant dynamics. Second-order theories that provide constraints in the form of necessary conditions may be considered true if they correctly rule out false first-order theories and false in that they incorrectly rule out true first-order theories.

In sum, I submit that Monton is grossly underestimating the argumentative work that would be necessary to brush SR to the side. Thus, he has failed, in my view, to sufficiently establish the first part of his argument, viz. to marginalize SR as an irrelevant and false theory. It turns out that in exactly those aspects which are relevant to a presentist, SR is too pertinacious to be so easily blown away by the simple need of a quantum theory of gravity. Let us turn to the second step of the argument.

\section{The CMC foliation approach: a new home for presentism?}

The constant mean curvature (CMC) foliation approach is a fixed-foliation theory as discussed in the previous section ${ }^{14}$ In fact - and this ends up undermining Monton's case - it is not really an approach to QG in its own right, but merely a technique that is explored on the road to QG. It starts out, like other canonical approaches to gravity, from a formulation of GR as a Hamiltonian system with constraints, dealing with spacetimes of topology $\Sigma \times \mathbb{R}$-in itself a limitation. The canonical variables are the 3-metric induced on the spacelike hypersurface $\Sigma$, which describes the geometry of $\Sigma$, and its extrinsic curvature, which specifies the embedding of $\Sigma$ in the four-dimensional manifold. The content of Einstein's field equations - the dynamical equations of the standard formulation of GR - is re-expressed in the constraint equations. These constraints define a subspace of the phase space $\Gamma$, the so-called constraint surface $\bar{\Gamma}$. In the CMC approach, only the subset $\Gamma_{\tau} \subset \bar{\Gamma}$ defined by the condition that the mean (i.e., the trace) of the extrinsic curvature is constant is considered. This mean (extrinsic) curvature is denoted by $\tau$. A spacelike hypersurface $\Sigma$ has constant mean curvature just in case $\tau$ is constant across $\Sigma$. Why does this condition deserve to be called a "time gauge", indicating that the spacetime is foliated into sets of "simultaneous" events? It just so turns out that a reasonably large open subset of the space of models of GR consist of spacetimes admitting a unique foliation into hypersurfaces parametrized by constant mean curvature. If a general-relativistic spacetime is sliceable into hypersurfaces of constant mean curvature - call these spacetimes CMC-sliceable-, then $\tau$ varies monotonically within a constant mean curvature foliation.

Starting out from the subset $\Gamma_{\tau} \subset \bar{\Gamma}$ of CMC-sliceable spacetimes, a particular foliation is chosen for every model in that subset: the CMC foliation. This move significantly reduces the

central tenets of modern physics" (241). Monton disagrees vehemently: He flatly denies that fixed-foliation approaches require a preferred coordinate system. He bases this denial on Kretschmann's objection to general covariance as a physically contentful constraint on theories. While it is perhaps true that fixed-foliation theories can all be formulated in a generally covariant manner, the objection becomes impotent if general covariance is interpreted in the correct, substantive way, i.e. as a gauge symmetry of GR. Although the particular formulation chosen by Belot and Earman may be unfortunate, their point essentially stands: fixed-foliation theories break the symmetry for which we have excellent reason to believe that every viable theory must respect it.

${ }^{14}$ This section is inevitably more technical than the rest of this essay, although an effort is made to provide a selfcontained characterization of the approach. For more extensive and rigorous presentations of the approach, consult Beig (1994, 74-77), Fischer and Moncrief (1997), Isenberg (1995), and Rendall (1996). Cf. also Belot and Earman (2001, particularly 239f). 
technical difficulty of solving the constraint equations in that it effectively eliminates three of the four usual constraint equations, and three of the four functions to be solved for. Essentially, reducing $\bar{\Gamma}$ to $\Gamma_{\tau}$ amounts to fixing the gauge, hence "time gauge". The only gauge freedom left are reparametrizations of $\tau$. Thus, general covariance is broken down to time-reparametrization invariance, which effectively brings the situation back to a time-reparametrization-invariant theory as characterized early in Section 2. Also, this step simplifies the remaining constraint equation to an equation linear in the momentum conjugate to $\tau$. Given a particular $\tau$-parametrization then, one can construct a Hamiltonian. The resulting time-dependent Hamiltonian $H(\tau)$ effectively measures the spatial volume of the universe. More precisely, it provides a measure for the volume of the Cauchy surface of mean extrinsic curvature $\tau$. Thus, as Beig $(1994,77)$ concludes, by selecting a distinguished parametrization, a time-dependent Hamiltonian system with the Hamiltonian given by the volume function can be constructed to mimic a cousin of GR. Once the classical Hamiltonian theory is in place, then, an attempt can be made at cooking it up into a quantum theory using the canonical recipe. It turns out that a canonical quantization of such a Hamiltonian system can successfully be completed for the $(2+1)$-dimensional cousin of GR, but not for the much more pertinent $(3+1)$-dimensional case of full GR.

The CMC approach has additional serious limitations, both at the classical and the quantum level. First, it is well-understood only for the vacuum case and for spatially closed spacetimes, i.e. for spacetimes with manifolds such that $\Sigma$ is compact and without boundary. There are good reasons to believe that the actual universe exemplifies neither of these properties. Second, not all globally hyperbolic, spatially closed vacuum spacetimes admit a foliation into hypersurfaces of constant mean curvature 15 Apart from the limitations noted above, this means that the CMC approach cannot deal with some general-relativistic spacetimes, even if we restrict those to be globally hyperbolic. There is no consensus as to how severe the restriction to globally hyperbolic spacetimes is. On the one hand, there are important classes of non-globally hyperbolic spacetimes ${ }^{16}$ On the other hand, important approaches to QG such as loop quantum gravity are confined to the same class of spacetimes. Also, the initial value problem can only meaningfully be addressed in the context of globally hyperbolic spacetimes. I will leave this question aside and instead turn to a brief discussion of the reach of the spacetimes amenable to a CMC-slicing.

Such a discussion starts out from the conformal reformulation of the standard constraint equations of Hamiltonian GR as proposed and developed by André Lichnerowicz and Yvonne ChoquetBruhat and James York (1980). The conformal method has proved to be a potent means to approach the Cauchy problem and has important applications in numerical GR. The question that is being asked is not which part of a given spacetime can be covered by a CMC foliation. Rather, the idea is to simultaneously construct or recover a full four-dimensional spacetime as the solution of a Cauchy problem as well as to obtain a CMC foliation of it, using the mean curvature $\tau$ to parametrize the foliation and thus to provide a global time function. Naturally, this approach cannot hope to result in anything other than globally hyperbolic spacetimes.

The approach starts out from initial data on a spacelike hypersurface $\Sigma$, the induced metric $\lambda_{a b}$ on $\Sigma$ and a symmetric tensor field $\sigma^{a b}$, which is trace-free $\left(\lambda_{a b} \sigma^{a b}=0\right)$ and divergence-free $\left({ }^{\lambda} \nabla_{a} \sigma^{a b}=0\right.$ where ${ }^{\lambda} \nabla$ is the covariant derivative compatible with $\left.\lambda_{a b}\right)$ with respect to $\lambda_{a b}$. The tensor field $\sigma^{a b}$ is the second fundamental form on $\Sigma$. Roughly, $\lambda$ corresponds to the spatial components of the metric and $\sigma$ to their time derivatives. To these, the scalar field $\tau$ is added. The triple $\left(\lambda_{a b}, \sigma^{a b}, \tau\right)$ on $\Sigma$, usually called the conformal data, then acts as initial data for the Hamiltonian equivalent of Einstein's field equations. This is to be understood in the sense that

\footnotetext{
${ }^{15}$ Cf. Bartnik (1988) and Rendall (1996).

${ }^{16} \mathrm{Cf}$. Smeenk and Wüthrich (forthcoming) for more on non-globally hyperbolic spacetimes.
} 
the usual initial data for the standard Hamiltonian field decomposition into induced 3-metrics and extrinsic curvature satisfy the standard constraint equations if and only if the conformal data satisfy the corresponding conformal constraint equations. These equations, which I am not going to reproduce here, constitute a coupled quasilinear elliptic system of partial differential equations that do not afford a solution for all choices of conformal data (and hence not for the corresponding standard situation). These equations pose formidable technical obstacles and do consequently not surrender to general solution. The reason why the CMC approach is pursued is because, as mentioned above, assuming that $\tau$ is constant - which is exactly what CMC does - offers a significant technical simplification at this point: It eliminates three of the four conformal constraint equations, as well as three of the four unknown functions to be solved for. The remaining constraint equation, often termed Lichnerowicz equation, although still not solved in the general case, permits the proving of theorems pertaining to the existence and uniqueness of solutions.

An important problem that arises in this context, the so-called Yamabe problem, is the issue of conformally rescaling a metric to obtain a metric of constant scalar curvature. It turns out that there is a solution to this problem for metrics on spatially compact manifolds. This is what the following theorem establishes (Isenberg 1995, 2252):

Theorem 1 (Yamabe) Let $\lambda_{a b}$ be a $C^{\infty}$ Riemannian metric on a closed three-dimensional manifold $\Sigma$. Then there exists a $C^{\infty}$ positive-definite function $\theta$ on $\Sigma$ such that the scalar curvature of the metric $\theta^{4} \lambda_{a b}$ is constant.

Yamabe's Theorem can be shown to imply, together with some propositions that require little extra work (ibid., 2253), that the set of all $C^{\infty}$ Riemannian metrics on $\Sigma$ can be partitioned into three Yamabe classes: Since each of these metrics will be conformal to a metric with constant scalar curvature 1,0 or -1, they fall exactly into one of the corresponding Yamabe classes denoted $\mathcal{Y}^{+}(\Sigma), \mathcal{Y}^{0}(\Sigma)$ or $\mathcal{Y}^{-}(\Sigma)$, respectively. For each $\lambda_{a b}$, its Yamabe class is thus a conformal invariant. It turns out that for some closed manifolds $\Sigma, \mathcal{Y}^{+}(\Sigma)$ and $\mathcal{Y}^{0}(\Sigma)$ are both empty, while $\mathcal{Y}^{-}(\Sigma)$ is never empty. Furthermore, $\mathcal{Y}^{0}(\Sigma)$ can only be empty if $\mathcal{Y}^{+}(\Sigma)$ is also empty, but the converse is not true.

James Isenberg (1995) systematically investigates for which sets of conformal data $\left(\lambda_{a b}, \sigma^{a b}, \tau\right)$ the Lichnerowicz equation can be solved and thus be mapped to a solution of the standard constraint equations, and for which sets it can't. As he shows, the solvability depends on three criteria. First, it depends on which Yamabe class $\lambda_{a b}$ belongs to. Second, it relies on whether $\sigma^{2}=\sigma_{a b} \sigma^{a b}$ is identically zero on $\Sigma$ or not. Finally, it matters whether the constant $\tau$ is zero or not. These criteria are all conformal invariants. Of the resulting twelve classes of conformal data, six map to solutions of the Lichnerowicz equation and six don't. More precisely, Isenberg $(1995,2259)$ shows the following theorem:

Theorem 2 (Isenberg) Let $\lambda_{a b}$ be a (sufficiently smooth) Riemannian metric on $\Sigma, \sigma_{a b}$ a symmetric tensor field on $\Sigma$ which is trace-free and divergence-free with respect to $\lambda_{a b}$, and $\tau$ a constant. Then the following table indicates for which conformal data $\left(\lambda_{a b}, \sigma^{a b}, \tau\right)$ the Lichnerowicz equation does ("Yes") or does not ("No") admit a solution:

$\begin{array}{ccccc} & \left(\sigma^{2} \equiv 0, \tau=0\right) & \left(\sigma^{2} \equiv 0, \tau \neq 0\right) & \left(\sigma^{2} \not \equiv 0, \tau=0\right) & \left(\sigma^{2} \not \equiv 0, \tau \neq 0\right) \\ \lambda_{a b} \in \mathcal{Y}^{+} & \text {No } & \text { No } & \text { Yes } & \text { Yes } \\ \lambda_{a b} \in \mathcal{Y}^{0} & Y e s & \text { No } & \text { No } & \text { Yes } \\ \lambda_{a b} \in \mathcal{Y}^{-} & \text {No } & \text { Yes } & \text { No } & \text { Yes }\end{array}$

For conformal data in the class $\left(\lambda_{a b} \in \mathcal{Y}^{0}, \sigma^{2} \equiv 0, \tau=0\right)$, the solution is non-unique; for all others the solution is unique if it exists. 
For any given closed three-manifold $\Sigma$, Isenberg's Theorem offers a "complete function space parametrization" (ibid.) of the set of CMC solutions of the standard constraints. In fact, the set of CMC solutions of the standard constraints stand in a one-to-one correspondence with what is essentially the direct sum of the six classes of conformal data as given in the table in Theorem 2 (ibid.) ${ }^{17}$

Before we press on to more pertinent matters, let me note the fact that for conformal data of the class $\left(\lambda_{a b} \in \mathcal{Y}^{0}, \sigma^{2} \equiv 0, \tau=0\right)$ (i.e., in case the metric is conformal to another one with vanishing scalar curvature everywhere on $\Sigma$, the square of the tensor field essentially giving its temporal derivative is identically zero on $\Sigma$, and the constant mean extrinsic curvature vanishes on $\Sigma$ ), we are confronted with a kind of indeterminism. Given conformal data of this category on $\Sigma$, there exist multiple solutions to the dynamical equations. In other words, for this class of field values on $\Sigma$, the initial state of the physical system does not, in tandem with the dynamical equations, uniquely determine the state of the physical system for all times. The construction of the conformal method does not yield a unique four-dimensional spacetime. It is appropriate to speak of indeterminism since $\Sigma$ can be considered a time slice on which the system's state is specified by the conformal data. From the fact that for a given folium with its constant mean curvature and initial data the dynamical development, and thus the construction of the full spacetime, is sometimes non-unique, it does not follow, as Earman $(2008,148)$ seems to suggest ${ }^{18}$ that for a given four-dimensional spacetime, its global foliation into hypersurfaces of constant mean curvature is sometimes non-unique, if it exists. The reason for this is that the different solutions will not correspond to different foliations of the same spacetime, but rather to different spacetimes altogether. Conversely, this in itself does not imply that CMC-slicings will be unique for a given spacetime, where they exist.

Be this as it may, the main problem of the CMC approach is, already at the classical level, that only a limited, although arguably important, class of spacetime models of GR comply in that they are CMC-sliceable. Unfortunately, as mentioned above, this class does not even exhaust the globally hyperbolic spacetimes of GR. Furthermore, also as stated above, it is only tractable for spatially closed vacuum spacetimes. But there is an additional difficulty, as pointed out by Isham (1991, 200): time-dependent Hamiltonians, as we find them here, have odd consequences. First, they are typically interpreted to mean, at least at the quantum level, that energy can enter or leave the quantum system, i.e., that the system is not closed. But this is odd indeed, as the system at stake is supposed to be the entire universe. Second, as Isham continues, for systems with timedependent Hamiltonians one cannot get the Wheeler-DeWitt equation for the reduced system from the relevant Schrödinger equation, which shows the inequivalence of different canonical approaches to QG. This may not ultimately amount to a strike against the CMC approach, but its advocate must find a way to accommodate this inequivalence.

One might dissent to using the CMC approach for presentist purposes with an analogue to Kurt Gödel's $(1949,562)$ objection to James Jeans's proposal to rest a robust notion of absolute time on the cosmological time of highly symmetrical spacetimes whose foliation into space and time sensitively depends on these symmetries. As Gödel insisted, whether or not absolute time existed should not depend on contingent matters of fact concerning the distribution of matter and energy in the actual universe. Similarly, a potential resuscitation of presentism by the CMC

\footnotetext{
17 "Essentially" because the space of conformal data must be quotiented out by the action of the group of conformal transformations, as well as by the action of the spatial diffeomorphism group in order for the correspondence to be one-to-one.

${ }^{18}$ When he writes that "[typically such a foliation is unique when it exists, but existence is guaranteed only for a limited class of solutions to Einstein's field equations, a class that does not exhaust solutions with causally nice features" (emphasis added). While I agree with every other part of the statement, I take issue with the first clause's suggestion that there may be cases where such foliation is not unique, for which I see no warrant in the literature.
} 
approach fails, the objection goes, on the grounds that the CMC foliation also depends on the same kinds of contingent facts. In defense of the CMC-inspired presentist, it should be noted, however, that the Gödel move is significantly weaker here than it was in the original case. The reason for this disanalogy is that CMC-sliceable spacetimes form, to repeat, open subsets in the space of solutions - unlike the highly symmetrical spacetimes relied on by Jeans. It is true that moving around the matter and energy content of the universe will in general deform the CMC foliation, but this will often not change the fact that there is a CMC foliation for the spacetime at stake.

Let us, for the sake of Monton's argument, ignore these limitations of the CMC approach and ask whether it would, if borne out, vindicate presentism, as Monton asserts. No, it would not; or at least not as easily as Monton seems to think. Apart from those limitations of the CMC approach already listed, it is far from clear whether the CMC approach can be exploited to underwrite a presentist metaphysics. In particular, it is far from obvious how the mean extrinsic curvature $\tau$ relates to physical time, despite the fact that it can be used as a global time parameter. What the presentist needs is an account of how $\tau$ gives raise to not just physical time, but a time that underwrites our presentist intuitions. The fact that the folia are Cauchy surfaces might help the presentist here, as this will permit to establish a direct connection to the initial value problem and issues of determinism, which, if anything, seem to be directly linked to the role of physical time 19 In the absence of such an account, a presentist such as Monton may rightly claim that the CMC approach, to the extent to which it is to be taken seriously as a fundamental, or at least true, physical theory, relieves the pressure that presentism has felt since the advent of SR. He has not yet, however, produced a positive argument in favour of presentism. For this, an account relating the CMC approach to our allegedly presentist phenomenology is essential.

Finally, lest the presentist gets overly enamoured of the CMC approach, it ought to be noted that no one takes it seriously as a physically plausible full theory of classical or quantum gravity. The real interest in the approach is fueled by the fact that it so significantly simplifies the systems of constraint equations that the Hamiltonian approach to GR is usually confronted with. Thus, the sole reason the CMC ansatz is explored is because it offers a technically tractable toy theory of canonical gravity 20 Overall, it is incapable of accommodating the full plethora of gravitational phenomena that a theory of gravity is expected to address. Finally, as a reminder, the irony that published work in the CMC approach has almost exclusively dealt with the classical level while Monton was really concerned with a fundamental quantum theory of gravity should not be lost on the reader.

\section{Conclusion}

Since there are no complete quantum theories of gravity available at present-let alone "theories of everything" - , the question of whether presentism is ultimately compatible with fundamental physics remains open. The most promising approaches to QG to date, string theory and loop quantum gravity, offer no respite for presentism. As far as I understand it, string theory is a fully Lorentz-invariant theory. Similarly, loop quantum gravity does not permit the introduction of preferred frames of reference and thus does not contain the resources to support a privileged foliation. As a matter of fact, there is a foreboding sense in which time evaporates completely as a fundamental physical magnitude in loop quantum gravity. Presumably, such physics could not underwrite Monton's project of reading a presentist metaphysics of time into the fundamental

\footnotetext{
${ }^{19}$ Although the potential non-uniqueness of CMC foliations would surely undermine such a connection if borne out.

${ }^{20}$ Cf. also Belot and Earman $(2001,241)$.
} 
physics ${ }^{21}$ Even on its own limited terms, I have argued that those approaches to QG that rely on fixed-foliations such as the CMC proposal are not as hospitable to presentism as Monton seems to think.

Let me conclude with Callender (2000) who warns against permitting presentism (or, more generally, any tensed theory of time) to "push us away from the traditional understanding of relativity" (S596), a role to be reserved for developments in physics. Monton shrugs this charge off by explaining that no non-traditional interpretation of relativity is required, since the presentist can simply deny that SR or GR are true theories just because they are incompatible with presentism. One man's modus ponens is truly another man's modus tollens. But if a re-interpretation of relativity against the backdrop of presentism is not warranted by evidence or argument, then the whole-sale rejection of it will hardly be more acceptable! Monton seems to think that, at least as viewed from a point of view of a committed presentist, since presentism is true, science should not, for its own good, turn out to be incompatible with it. Since alternative approaches to QG are incompatible with becoming, and since the existence of becoming is a philosophical, not a scientific, issue for the presentist, "we should expect the correct theory of quantum gravity to be a fixed foliation theory" (op. cit., 274). But that's exactly the point: if we base the scientific decision among competing theories on metaphysical predilections, we better have good reasons to do so. A failure to appreciate this would mislead us into abandoning Naturalism, or anyway naturalism. 22

In this vein, Callender continues by asking, quite pertinently in my view, "if science cannot find the 'becoming frame', what extra-scientific reason is there for positing it?" (S597) Monton (op. cit., 272) replies to this charge by insisting that he can't discern a reason why the presentist ought to be committed to the antecedent. The grounds for denying the antecedent of Callender's conditional statement, Monton believes, can be found in that the CMC foliation approach yields what can be interpreted as the becoming frame. To be sure, we would need some sort of account of how exactly the CMC foliation of a spacetime underwrites "becoming" for that move to be successful. Monton recognizes that he cannot offer any positive account from our experiences to the necessity of the becoming frame, or of how the becoming frame is coupled to a CMC foliation, but he defends himself by retorting that "just because we do not have a good argument for the presentist doctrine... does not mean that the doctrine is false" (ibid., 273n). True, but in the absence of such argument, there is little or no reason to take the CMC foliation approach seriously as a full-fledged physical theory potent enough to supplant GR. As we have seen, this approach is highly limited in its applicability, remains almost exclusively at the classical level, and does not offer a viable road to a resuscitation of presentism. More seriously still, if what I said above is true, then we do have reason to accept the antecedent of Callender's pronouncement.

If we accept the antecedent, however, then the presentist must give sound arguments that are sufficiently forceful to overturn time-honoured Lorentz invariance as a constraint on a future theory of QG. That does not seem to be forthcoming. On balance, I submit, the prospects of presentism look rather dim.

\footnotetext{
${ }^{21}$ Monton (op. cit., 277) thinks that the presentist can evade the problem of time by simply maintaining that the position does not speak to fundamental reality, but only to time. Thus, if time is emergent rather than fundamental, presentism would be true as long as the emergent time fits the presentist metaphysics. While I acknowledge this possibility, it doesn't offer an appealing option to the presentist.

${ }^{22}$ Monton recognizes this possibility when he offers an alternative move: the presentist could decide to give up scientific, but not metaphysical, realism.
} 


\section{Acknowledgements}

I am indebted to Craig Callender, Jonathan Cohen, John Earman, Storrs McCall, Bradley Monton, Thomas Müller, Vesselin Petkov, and Steve Savitt for discussions and comments, and audiences in Montreal and Geneva for their engagement with this paper. I also thank Vesselin Petkov for his almost infinite patience with my procrastination. This project has been funded in part by the Swiss National Science Foundation ("Properties and Relations", 100011-113688), by the University of California, San Diego, and by the Hellman Family Foundation.

\section{References}

[1] Amelino-Camelia, Giovanni, "Quantum gravity phenomenology", available online at http://arxiv.org/abs/0806.0339 (2008).

[2] Barbour, Julian, The End of Time: The Next Revolution in Physics, Oxford: Oxford University Press (1999).

[3] Bartnik, Robert, "Remarks on cosmological spacetimes and constant mean curvature surface", Communications in Mathematical Physics 117 (1988): 615-624.

[4] Beig, Robert, "The classical theory of canonical general relativity", Lecture Notes in Physics 434 (1994): 59-80.

[5] Belot, Gordon and John Earman, "Pre-Socratic quantum gravity", in Craig Callender and Nick Huggett (eds.), Physics Meets Philosophy at the Planck Scale, Cambridge: Cambridge University Press (2001), 213-255.

[6] Callender, Craig, "Shedding light on time", Philosophy of Science 67 (2000): S587-S599.

[7] Callender, Craig, "Finding 'real' time in quantum mechanics", in William L Craig and Quentin Smith (eds.), Einstein, Relativity, and Absolute Simultaneity, London: Routledge (2008), 5072 .

[8] Choquet-Bruhat, Yvonne and James W York, "The Cauchy problem", in Alan Held (ed.), General Relativity and Gravitation: One Hundred Years After the Birth of Albert Einstein, Volume 1, New York: Plenum (1980), 99-172.

[9] Craig, William L, Time and the Metaphysics of Relativity, Dordrecht: Kluwer Academic Publishers (2001).

[10] Dieks, Dennis (ed.), The Ontology of Spacetime, Amsterdam: Elsevier (2006).

[11] Dorato, Mauro, "The irrelevance of the presentist/eternalist debate for the ontology of Minkowski spacetime", in Dieks (2006), 93-109.

[12] Earman, John, "Reassessing the prospects for a growing block model of the universe", International Studies in the Philosophy of Science 22 (2008): 135-164.

[13] Fine, Kit, "Tense and reality", in his Modality and Tense: Philosophical Papers, Oxford: Oxford University Press (2005), 261-320.

[14] Fischer, Arthur E and Vincent Moncrief, "Hamiltonian reduction of Einstein's equations of general relativity", Nuclear Physics B (Proc. Suppl.) 57 (1997): 142-161. 
[15] Gödel, Kurt, "A remark about the relationship between relativity theory and idealistic philosophy", in Paul A Schilpp (ed.), Albert Einstein: Philosopher-Scientist, New York: Tudor (1949), 557-562.

[16] Harrington, James, "Special relativity and the future: a defense of the point present", Studies in History and Philosophy of Modern Physics 39 (2008): 82-101.

[17] Hinchliff, Mark, "The puzzle of change", Philosophical Perspectives 10 (1996): 119-136.

[18] Isenberg, James, "Constant mean curvature solutions of the Einstein constraint equations on closed manifolds", Classical and Quantum Gravity 12 (1995): 2249-2274.

[19] Isham, Christopher J, "Conceptual and geometrical problems in quantum gravity", Lecture Notes in Physics 396 (1991): 123-229.

[20] McCall, Storrs, "QM and STR", Philosophy of Science 67 (2000): S535-S548.

[21] Monton, Bradley, "Presentism and quantum gravity", in Dieks (2006), 263-280.

[22] Putnam, Hilary, "Time and physical geometry", Journal of Philosophy 64 (1967): 240-247.

[23] Rendall, Alan D, "Constant mean curvature foliations in cosmological spacetimes", Helvetica Physica Acta 69 (1996): 490-500.

[24] Rietdijk, C Wim, "A rigorous proof of determinism derived from the special theory of relativity", Philosophy of Science 33 (1966): 341-344.

[25] Savitt, Steven F, "Presentism and eternalism in perspective", in Dieks (2006a), 111-127.

[26] Savitt, Steven, "Being and becoming in modern physics", in Edward N Zalta (ed.), Stanford Encyclopedia of Philosophy, available online at http://plato.stanford.edu/entries/spacetimebebecome/ (2006b).

[27] Smeenk, Christopher and Christian Wüthrich, "Time travel and time machines", in Craig Callender (ed.), The Oxford Handbook of Time, Oxford: Oxford University Press (forthcoming).

[28] Stein, Howard, "On relativity theory and openness of the future", Philosophy of Science $\mathbf{5 8}$ (1991): 147-167.

[29] Tooley, Michael, Time, Tense, $\&$ Causation, Oxford: Oxford University Press (1997).

[30] Will, Clifford M, "Was Einstein right? Testing relativity at the centenary", in Abhay Ashtekar (ed.), 100 Years of Relativity; Space-Time Structure: Einstein and Beyond, Singapore: World Scientific (2005a), 205-227.

[31] Will, Clifford M, "Special relativity: A centenary perspective", Séminaire Poincaré 1 (2005b): 79-98.

[32] Wüthrich, Christian, "Demarcating presentism", manuscript (unpublished).

[33] Zimmerman, Dean, "The privileged present: defending an 'A-theory' of time", in Theodore Sider, John Hawthorne, and Dean Zimmerman (eds.), Contemporary Debates in Metaphysics, Malden, MA: Blackwell (2008), 211-225. 